Manuscript Type: Brief article

\title{
Neoadjuvant treatment in young women with breast cancer
}

Agustí Barnadas $^{1}$

${ }^{1}$ Hospital de Santa Creu i Sant Pau, Barcelona, Spain.

Corresponding author:

Agustí Barnadas, MD

Department of Medical Oncology

Hospital de Santa Creu i Sant Pau

Calle Sant Antoni Maria Claret, 167

08025-Barcelona. Spain

E-mail: $\quad$ abarnadas@santpau.cat

Phone: $\quad$ +34 935565638

Fax: $\quad$ +34 $\quad$ 335565769 


\section{Introduction}

Primary or neoadjuvant chemotherapy was initially developed for the management of young women with breast cancer for whom surgery, either radical or conservation, was not appropriate, offering the possibility of mammary gland excision to a large proportion of patients with previously inoperable tumors. Clinical characteristics of these patients included tumors greater than $5 \mathrm{~cm}$ (T3), fixation of the tumor to the skin or chest wall (T4), skin edema, skin ulceration, satellite nodules or infiltration, large $(>2.5 \mathrm{~cm})$ or fixed/matted axillary lymph nodes (N2), supraclavicular lymphadenopathy or arm edema [1]. Among the main reasons for avoiding surgical treatment as the primary approach in this group of patients is that local disease recurs in more than $50 \%$ of patients, and only $13-20 \%$ survive more than five years [2].

Multiple large randomized trials have proven the safety of neoadjuvant chemotherapy, with comparable efficacy to adjuvant chemotherapy regarding disease-free survival (DFS) and overall disease progression. Neoadjuvant chemotherapy leads to complete clinical response in $10-30 \%$ of patients, and partial responses in $50-60 \%$ [3-5]. In addition, it offers the opportunity to assess in vivo chemosensitivity of breast tumors and eventually to correlate tumor response with long-term outcome [6]. Moreover, primary chemotherapy allows a greater proportion of women to benefit from conservative surgery, instead of having to resort to a more radical approach.

Unfortunately, there are no prospective studies on neoadjuvant chemotherapy in young breast cancer patients, and therefore decisions must be inferred from results obtained from completed or ongoing trials on the general population. However, there is a general agreement on some important aspects in this age group that should be taken into account when choosing the therapeutic regimen, such as the wish to preserve the breast, the need to minimize adverse events, and the preservation of reproductive function in women of childbearing age. 


\section{Therapeutic options in neoadjuvant chemotherapy}

The largest randomized studies comparing neoadjuvant versus adjuvant chemotherapy are the National Surgical Adjuvant Breast and Bowel Projects (NSABP) B-18 and B-27 $[5,7]$. In trial B-18, 1523 patients with operable, palpable breast cancer were randomly assigned to receive doxorubicin and cyclophosphamide therapy either as neoadjuvant or adjuvant chemotherapy. After a median of 16 years of follow-up, the results showed no statistically significant differences in DFS and overall survival (OS) between the two groups. However, there was a trend in favor of neoadjuvant chemotherapy in these two parameters for women less than 50 years old ( $p=0.09$ for DFS and $p=0.06$ for OS) [5]. Hence, although there is no survival benefit from neoadjuvant chemotherapy, it can be considered as effective as adjuvant chemotherapy, and allows more breast conserving therapies.

In trial NSABP B-27, 2411 women with similar characteristics were randomized to receive neoadjuvant doxorubicin and cyclophosphamide. In addition, one of the three groups of patients received neoadjuvant docetaxel preoperatively. The results after 16 years of follow-up demonstrated that the addition of docetaxel to doxorubicin and cyclophosphamide did not have a significant impact on DFS or OS. However, when docetaxel was added to doxorubicin and cyclophosphamide before surgery, a significantly higher proportion of patients with pathologic complete response $(\mathrm{pCR})$ was observed in comparison with neoadjuvant doxorubicin and cyclophosphamide alone (26\% vs $13 \%$ respectively; $p<0.0001)$ [5].

Recently, the results of a trial in 1355 women with operable breast cancer evaluating the efficacy of adding paclitaxel to doxorubicin followed by cyclophosphamide, methotrexate and fluorouracil (CMF) in the adjuvant setting, and the comparison of this regimen with the same one given as primary therapy, showed similar results in terms of relapse-free survival $(p=0.18)$. However, similarly to the results observed in the NSABP 
B-18 trial, the rate of breast-conserving surgery was significantly higher $(p<0.001)$ in the neoadjuvant arm [8].

In summary, given the encouraging results obtained with taxane or anthracyclinebased regimens in the neoadjuvant setting of breast cancer, at present it is accepted that the management of young women with early breast cancer should include both agents.

\section{Recommended dosage of neoadjuvant chemotherapy}

Results of a recent study comparing preoperative intense dose-dense (IDD) and doseescalated sequential epirubicin followed by paclitaxel every 2 weeks with concurrent preoperative epirubicin plus paclitaxel every 3 weeks showed that neoadjuvant IDD treatment significantly improved pCR $(p=0.008)$, DFS $(p=0.011)$ and OS $(p=0.041)$ compared with concurrent preoperative epirubicin plus paclitaxel [9]. Importantly, between $45-52 \%$ of the study population were women aged less than 50 years old. However, results from other studies have not been so promising in terms of DFS and OS [10]. Thus, the decision to increase dose intensity in young breast cancer patients has not been conclusively resolved and awaits confirmation from ongoing studies.

\section{Role of the selective sentinel node biopsy (SNB)}

The degree of nodal involvement is the most significant prognostic factor in patients with early-stage cancer. Besides being the most accurate method of assessing tumor spread to the lymph nodes, SNB allows staging of the disease and prognostic assessment, and guides treatment selection [11]. Nevertheless, to date there is no combination of predictors that can replace surgical resection and histopathologic examination of the sentinel lymph node or, in case of positivity, all the removed lymph nodes when axillary lymph node dissection (ALND) is performed. 
In study NSABP B-27, so far the largest retrospective review of patients with operable breast cancer, SNB was performed on 428 patients (18\%) before the required ALND. At least one sentinel lymph node was identified and removed in 363 patients (success rate, $85 \%$ ); overall accuracy was $96 \%$, sensitivity was $89 \%$ and negative predictive value was $93 \%$. Among patients who had pathologically positive nodes, the falsenegative rate was $11 \%$ [12].

Nevertheless, the American Society of Clinical Oncology guidelines state that, although technically feasible, there is insufficient evidence to recommend the use of SNB after neoadjuvant chemotherapy, and limited evidence to recommend its use before neoadjuvant chemotherapy. Therefore, it is concluded that SNB should only be performed in the setting of clinically negative axillary lymph nodes [11].

\section{Role of neoadjuvant radiotherapy}

Radiotherapy is an integral part of the loco-regional management of locally advanced breast cancer, and in patients with persistent residual disease following breast conservation surgery. In operable tumors the use of boost radiation after conservative surgery is well established, especially in young women. However, the issue of administering radiotherapy alone, instead of surgery, after neoadjuvant chemotherapy is controversial [2]. In a study carried out by the Cancer and Leukemia Group B (CALGB), 81 patients were randomized to either surgery or radiotherapy after having received three months of neoadjuvant chemotherapy. After a median follow up of 37 months, there were no significant differences in either DFS or OS [2]. Indirect comparisons between this and other trials suggest that local control rates are higher when both surgery and radiotherapy are used [2]. Adjuvant radiotherapy is also recommended after obtaining a pCR. 


\section{Prognostic factors}

Prognostic factors help clinicians to predict what the clinical outcome in these patients will be. There is no doubt that the best prognostic factor in young patients with breast cancer is achieving pCR. Studies on neoadjuvant chemotherapy have shown that patients who achieve $\mathrm{pCR}$ have significantly superior DFS and OS outcomes compared with patients who do not [5]. The tumor's molecular subtype may also be used to predict response to neoadjuvant chemotherapy. For example, the basal-like and human epidermal growth factor receptor-2 (HER-2) positive subtypes of breast cancer are more sensitive to paclitaxel- and doxorubicin-based neoadjuvant chemotherapies than luminal and normal-like tumors [13]. Finally, specific geneexpression signatures have also been shown to predict pCR following neoadjuvant chemotherapy with an extremely high overall accuracy, sensitivity and specificity [14]. In the near future, the routine use of gene expression profiling is expected to improve the selection of patients for neoadjuvant chemotherapy.

\section{Role of targeted therapies}

The identification of molecular targets has stimulated a growing interest in the use of targeted therapies in the neoadjuvant setting. To date, the largest study evaluating the use of one of these targeted therapies, namely trastuzumab, is the NeOAdjuvant Herceptin (NOAH) trial. Preliminary results of this study showed that patients with locally advanced HER2-positive breast cancer had a significant improvement in eventfree survival when trastuzumab was added to neoadjuvant chemotherapy. Additionally, almost twice as many trastuzumab-treated patients achieved pCR in comparison with the control arm of the study [15].

Other trials on targeted therapies in the neoadjuvant setting, such as the trial developed by the Grupo Español de Investigación del Cáncer de Mama (GEICAM) 
2006-03, GEICAM 2006-014, CALGB 40603 and NSABP B-40 are currently ongoing (www.clinicaltrials.gov).

\section{Conclusions}

Neoadjuvant therapy should be considered for young women with operable tumors in whom breast conserving surgery cannot be performed. Currently, the best predictor of therapeutic efficacy is $\mathrm{pCR}$, therefore all efforts should be aimed at increasing that. Advances in the identification of molecular tumor types should allow tailoring of treatments to each patient's needs. To date, this objective has been achieved with trastuzumab in combination with standard neoadjuvant chemotherapy. There are still several unresolved issues in the treatment of young women, such as whether to carry out SNB or ALND, whether loco-regional therapy should be administered to patients with $\mathrm{pCR}$ or whether systemic therapy should be administered to patients with residual tumor after neoadjuvant treatment, which await the results from ongoing studies. 


\section{Acknowledgements}

The authors acknowledge the support of Pfizer Spain, which facilitated the necessary meetings to evaluate and discuss all the data presented in this review, and Dr. Ximena Alvira from HealthCo SL (Madrid, Spain) for assistance in the preparation of this manuscript. 


\section{References}

1. Giordano SH (2003) Update on Locally Advanced Breast Cancer. Oncologist 8:(6):521-530

2. Rustogi A, Budrukkar A, Dinshaw K, Jalali R (2005) Management of locally advanced breast cancer: evolution and current practice. J Cancer Res Ther $1:(1): 21-30$

3. Buchholz TA, Lehman CD, Harris JR, Pockaj BA, Khouri N, Hylton NF, et al. (2008) Statement of the science concerning locoregional treatments after preoperative chemotherapy for breast cancer: a National Cancer Institute conference. J Clin Oncol 26:(5):791-7

4. Mauri D, Pavlidis N, loannidis JP (2005) Neoadjuvant versus adjuvant systemic treatment in breast cancer: a meta-analysis. J Natl Cancer Inst 97:(3):188-94

5. Rastogi P, Anderson SJ, Bear HD, Geyer CE, Kahlenberg MS, Robidoux A, et al. (2008) Preoperative chemotherapy: updates of National Surgical Adjuvant Breast and Bowel Project Protocols B-18 and B-27. J Clin Oncol 26:(5):778-85

6. Fisher B, Mamounas EP (1995) Preoperative chemotherapy: a model for studying the biology and therapy of primary breast cancer. J Clin Oncol 13:(3):537-40

7. Wolmark N, Wang J, Mamounas E, Bryant J, Fisher B (2001) Preoperative chemotherapy in patients with operable breast cancer: nine-year results from National Surgical Adjuvant Breast and Bowel Project B-18. J Natl Cancer Inst Monogr (30):96-102

8. Gianni L, Baselga J, Eiermann W, Porta VG, Semiglazov V, Lluch A, et al. (2009) Phase III trial evaluating the addition of paclitaxel to doxorubicin followed by cyclophosphamide, methotrexate, and fluorouracil, as adjuvant or primary systemic therapy: European Cooperative Trial in Operable Breast Cancer. J Clin Oncol 27:(15):2474-81 
9. Untch M, Mobus V, Kuhn W, Muck BR, Thomssen C, Bauerfeind I, et al. (2009) Intensive Dose-Dense Compared With Conventionally Scheduled Preoperative Chemotherapy for High-Risk Primary Breast Cancer. J Clin Oncol 27:(18):29382945

10. Del Mastro L, Dozin B, Aitini E, Catzeddu T, Baldini E, Contu A, et al. (2008) Timing of adjuvant chemotherapy and tamoxifen in women with breast cancer: findings from two consecutive trials of Gruppo Oncologico Nord-OvestMammella Intergruppo (GONO-MIG) Group. Ann Oncol 19:(2):299-307

11. Lyman GH, Giuliano AE, Somerfield MR, Benson AB, III, Bodurka DC, Burstein HJ, et al. (2005) American Society of Clinical Oncology Guideline Recommendations for Sentinel Lymph Node Biopsy in Early-Stage Breast Cancer. J Clin Oncol 23:(30):7703-7720

12. Mamounas EP, Brown A, Anderson S, Smith R, Julian T, Miller B, et al. (2005) Sentinel Node Biopsy After Neoadjuvant Chemotherapy in Breast Cancer: Results From National Surgical Adjuvant Breast and Bowel Project Protocol B27. J Clin Oncol 23:(12):2694-2702

13. Rouzier R, Perou CM, Symmans WF, Ibrahim N, Cristofanilli M, Anderson K, et al. (2005) Breast cancer molecular subtypes respond differently to preoperative chemotherapy. Clin Cancer Res 11:(16):5678-85

14. Thuerigen O, Schneeweiss A, Toedt G, Warnat P, Hahn M, Kramer H, et al. (2006) Gene expression signature predicting pathologic complete response with gemcitabine, epirubicin, and docetaxel in primary breast cancer. J Clin Oncol 24:(12):1839-45

15. Gianni L, Eiermann W, Semiglazov V, Manikhas A, Lluch A, Tjulandin S, et al. (2010) Neoadjuvant chemotherapy with trastuzumab followed by adjuvant trastuzumab versus neoadjuvant chemotherapy alone, in patients with HER2positive locally advanced breast cancer (the NOAH trial): a randomised 
controlled superiority trial with a parallel HER2-negative cohort. Lancet 375:(9712):377-84 